\title{
ON THE CONVERGENCE OF CERTAIN TRIGONOMETRIC
}

\section{AND POLYNOMIAL APPROXIMATIONS*}

\author{
BY
}

\section{DUNHAM JACKSON}

1. Introduction. Let $f(x)$ be a continuous function of period $2 \pi$. Let $T_{m n}(x)$ be the trigonometric sum of order $n$ or lower for which the integral

$$
\int_{0}^{2 \pi}\left|f(x)-T_{m n}(x)\right|^{m} d x
$$

has the smallest possible value, when $m$ and $n$ are given. The writer has recently proved the existence and uniqueness of $T_{m n}(x)$ for any positive integral value of $n$ and for any value of $m \geqq 1$, whether integral or not, $\dagger$ and has given some indication of the behavior of $T_{m n}(x)$, for fixed $n$, as $m$ becomes infinite. +

The first purpose of the present paper is to discuss the convergence of $T_{m n}(x)$ toward the value $f(x)$, when $m$ is held fast and $n$ is allowed to become infinite. It is found to be a sufficient condition for uniform convergence that $\lim _{\delta=0} \omega(\delta) / \sqrt[m]{\delta}=0$, where $\omega(\delta)$ is the modulus of continuity $\S$ of the function $f(x)$, that is, the maximum of $\left|f\left(x^{\prime}\right)-f\left(x^{\prime \prime}\right)\right|$ for $\left|x^{\prime}-x^{\prime \prime}\right| \leqq \delta$. The proof makes use of Bernstein's theorem on the derivative of a trigonometric sum. For $m=1$, the condition as stated would require that $f(x)$ be a constant, but in this case it is sufficient that $f(x)$ have a continuous derivative.

One would be inclined to expect that convergence would be more likely for large than for small values of $m$, and the result obtained is favorable to this view, as far as it goes. The theorem is less general, however, even for large

\footnotetext{
* Presented to the Society, September 8 and October 30, 1920.

† For $m>1$, see D. Jackson, On functions of closest approximation, these T r a n s a ct i o s s, vol. 22 (1921), pp. 117-128; for $m=1$, D. Jackson, Note on a class of polynomials of approximation, submitted to these $\mathrm{Transact}$ i o $\mathrm{ns}$. These papers will be referred to by the letters $A$ and $B$ respectively. In $A$, the treatment applies to a general class of approximating functions, including trigonometric sums as a very special case; in $B$, the discussion relates primarily to polynomials, but attention is called to the fact that the case of trigonometric sums is to be dealt with in the same way.

$\ddagger$ Loc. cit. A, $888,9$.

For the name, cf. de la Vallée Poussin, Leçons sur l'approximation des fonctions d'une variable rélle, Paris, 1919, pp. 7-8.
} 
values of $m$, than the well-known Lipschitz-Dini condition in the case of Fourier's series, $m=2$, and the present results may for this reason be regarded as only preliminary

The latter part of the paper contains a discussion of the corresponding problem in polynomial approximation. The method of treatment is generally the same, though the details of the reasoning and the conclusions reached are not exactly parallel in the two cases.

To return to the trigonometric case, it will be supposed throughout the discussion that $m$ has a fixed value $\geqq 1$, given in advance. For the sake of simplicity, the subscript $m$ will be omitted, and $T_{m n}(x)$ will be denoted simply by $T_{n}(x)$. This particular trigonometric sum will be referred to briefly as the approximating function of order $n$.

2. Reduction of the problem. If $T_{n}(x)$ is the approximating function for $f(x)$, and $t_{n}(x)$ is an arbitrary trigonometric sum of order $n$ or lower, the approximating function for $f(x)+t_{n}(x)$ will be $T_{n}(x)+t_{n}(x)$. For the error is identically the same in both cases, and the integral of the $m$ th power of its absolute value is the same, and if it were possible to diminish the value of the integral in one case, it could be diminished in the other case as well.*

Suppose there exists a trigonometric sum $t_{n}(x)$ such that

$$
\left|f(x)-t_{n}(x)\right| \leqq \epsilon
$$

for all values of $x$, where $\epsilon$ is some small positive quantity. Let

$$
\phi(x)=f(x)-t_{n}(x) .
$$

Then the error of the approximating function for $\phi(x)$ is the same as the error of the approximating function for $f(x)$, and the problem of determining the magnitude of the error in the case of $f$ is reduced to the corresponding problem for $\phi$.

3. Relation between maximum and integral. Let $\epsilon$ be an arbitrary positive quantity; let $\phi(x)$ be an arbitrary continuous function of period $2 \pi$, subject to the condition that

$$
|\phi(x)| \leqq \epsilon
$$

for all values of $x$; and let $t(x)$ be an arbitrary trigonometric sum of order not exceeding $n$. Throughout this section, $n$ will be fixed in value, and need not be indicated as a subscript. Let $\mu$ be the maximum of $|t(x)|$, and let $x_{0}$ be a point such that $\left|t\left(x_{0}\right)\right|=\mu$. Finally, let it be supposed that $\mu \geqq 4 \epsilon$. If $\mu<4 \epsilon$, this fact in itself will serve the purpose for which the conclusion of the present section is to be used in the contrary case.

* The relation between given function and approximating function is of course not linear in general; that is, the approximating function for the sum of two given functions is not generally the sum of the approximating functions. 
A theorem of S. Bernstein,* the importance of which is only beginning to be realized, states that the maximum of the absolute value of the derivative of a trigonometric sum of order $n$ can not exceed $n$ times the maximum of the absolute value of the original sum itself. That is, in the present case,

for all values of $x$. When

$$
\left|t^{\prime}(x)\right| \leqq \mu n
$$

therefore,

$$
\left|x-x_{0}\right| \leqq \frac{1}{2 n}
$$

$$
\left|t(x)-t\left(x_{0}\right)\right| \leqq \frac{1}{2} \mu,
$$

by the mean value theorem, and

$$
|t(x)| \geqq \frac{1}{2} \mu \text {. }
$$

Since, furthermore, $|\phi(x)| \leqq \epsilon$ and $\epsilon \leqq \mu / 4$,

$$
|t(x)-\phi(x)| \geqq \frac{1}{4} \mu
$$

throughout the interval (1), of length $1 / n$. From this it follows that

$$
\int_{0}^{2 \pi}|t(x)-\phi(x)|^{m} d x \geqq \frac{1}{n} \cdot\left(\frac{\mu}{4}\right)^{m} .
$$

4. Proof of convergence. Now let $\tau_{n}(x)$ be the approximating function of order $n$ for $\phi(x)$, let $\mu_{n}$ be the maximum of $\left|\tau_{n}(x)\right|$, and let

$$
\gamma_{n}=\int_{0}^{2 \pi}\left|\tau_{n}(x)-\phi(x)\right|^{m} d x
$$

If $\tau_{n}(x)$ were replaced by zero in the last integral, the value of the integral would be less than or equal to $2 \pi \epsilon^{m}$, since $|\phi| \leqq \epsilon$. But $\tau_{n}(x)$ has the property of giving the integral its minimum value, and so it is certain that

$$
\gamma_{n} \leqq 2 \pi \epsilon^{m} \text {. }
$$

By the preceding section, on the other hand, if $\mu_{n} \geqq 4 \epsilon$,

So

$$
\gamma_{n} \geqq \frac{1}{n} \cdot\left(\frac{\mu_{n}}{4}\right)^{m}
$$

$$
\begin{aligned}
\frac{1}{n} \cdot\left(\frac{\mu_{n}}{4}\right)^{m} & \leqq 2 \pi \epsilon^{m}, \\
\mu_{n} & \leqq 4 \epsilon(2 \pi)^{1 / m} n^{1 / m} .
\end{aligned}
$$

* See, e.g., de la Vallée Poussin, op. cit., pp. 39-42; also S. Bernstein, Sur l'ordre de la meilleure approximation des fonctions continues par des polynomes de degré donné, Mémoire couronné, Brussels, 1912, p. 20; and de la Vallée Poussin, C o m p t e s R e n d u s, vol. 166 (1918), pp. 843-846. In Bernstein's original statement of the theorem, the factor introduced on differentiating was $2 n$, instead of $n$. 
But if $\mu_{n}<4 \epsilon$, the last inequality is satisfied $a$ fortiori, and the relation is therefore true generally. To summarize:

If $\phi(x)$ is a continuous function of period $2 \pi$ which never exceeds $\epsilon$ in absolute value, $\tau_{n}(x)$ the approximating function of order $n$ for $\phi(x)$, and $\mu_{n}$ the maximum of $\left|\tau_{n}(x)\right|$, then

$$
\mu_{n} \leqq k_{m} n^{1 / m} \epsilon,
$$

where $k_{m}$ is a constant depending only on $m$.

Let $\rho_{n}$ be the maximum of $\left|\tau_{n}(x)-\phi(x)\right|$; then $\rho_{n}$ satisfies an inequality of the same form as $\mu_{n}$. For $\rho_{n} \leqq \mu_{n}+\epsilon$, and so

$$
\rho_{n} \leqq\left(k_{m}+1\right) n^{1 / m} \epsilon \text {. }
$$

In consequence of $\$ 2$, the last assertion has the following further significance: If $f(x)$ is a continuous function of period $2 \pi$ which can be represented by a trigonometric sum of order $n$ or lower with an error not exceeding $\epsilon_{n}$, if $T_{n}(x)$ is the approximating function of order $n$ for $f(x)$, corresponding to the exponent $m$, and if $\rho_{n}$ is the maximum of $\left|f(x)-T_{n}(x)\right|$, then

$$
\rho_{n} \leqq\left(k_{m}+1\right) n^{1 / m} \epsilon_{n},
$$

where $k_{m}$ has the same meaning as before.

Hence $T_{n}(x)$ will converge uniformly to the value $f(x)$, if $f(x)$ can be represented by trigonometric sums for successive values of $n$ in such a way that

$$
\lim _{n=\infty} \sqrt[n]{n} \cdot \epsilon_{n}=0
$$

Let $\omega(\delta)$ be the maximum of $\left|f\left(x^{\prime}\right)-f\left(x^{\prime \prime}\right)\right|$ for $\left|x^{\prime}-x^{\prime \prime}\right| \leqq \delta$. Then there will exist representations* of $f(x)$ such that $\epsilon_{n} \leqq c \omega(2 \pi / n)$, where $c$ is independent of $n$, for all positive integral values of $n$. If

$$
\lim _{\delta=0} \omega(\delta) / \sqrt[m]{\delta}=0
$$

then $\sqrt[m]{n / 2 \pi} \cdot \omega(2 \pi / n)$ will approach zero as $n$ becomes infinite, and the condition for convergence will be satisfied.

In the case $m=1$, the condition $\lim _{\delta=0} \omega(\delta) / \delta=0$ would require that $f(x)$ be a constant. There will exist representations $\dagger$ of $f(x)$ such that

$$
\lim _{n=\infty} n \epsilon_{n}=0,
$$

however, provided that $f(x)$ has a continuous derivative, so that the last-named condition is sufficient for convergence in this case.

If $\omega(\delta)$ approaches zero more rapidly than is required by the criterion for

* Cf., e.g., D. Jackson, On the approximate representation of an indefinite integral, etc., these Trans a c t i o s s, vol. 14 (1913), pp. 343-364; p. 350.

$\dagger$ Cf., e.g., the paper cited in the preceding footnote, pp. 350-351.

Trans. Am. Math. Soc. 11 
convergence, the relation (2), together with such general theorems on trigonometric approximation as are contained in the paper just cited, will give information with regard to the degree of convergence of the present approximating functions.

It may be remarked that the reasoning above, as applied to the case $m=2$, gives a new proof of the convergence of the Fourier's series for an extensive class of functions, although, in view of its dependence on Bernstein's theorem and on the general theory of approximation by trigonometric sums, the proof is not more elementary than others that are well known.

5. Polynomial case, end-points included. The theorem on the derivative of a trigonometric sum, on which the preceding proof is based, has a counterpart relating to polynomials. Let $P_{n}(x)$ be a polynomial of the $n$th degree or lower, such that $\left|P_{n}(x)\right| \leqq \mu$ for $-1 \leqq x \leqq 1$, where $\mu$ is a constant. Let $x=\cos \theta$; then $P_{n}(x)$ is a trigonometric sum in $\theta$, of order $n$ or lower, the absolute value of which is $\leqq \mu$ for all values of $\theta$. By the theorem for the trigonometric case,

That is, ${ }^{*}$

$$
\left|\frac{d}{d \theta} P_{n}(\cos \theta)\right|=\left|\sin \theta P_{n}^{\prime}(\cos \theta)\right| \leqq \mu n .
$$

for $-1 \leqq x \leqq 1$

$$
\left|\sqrt{1-x^{2}} P_{n}^{\prime}(x)\right| \leqq \mu n
$$

By means of a linear transformation on $x$, it can be inferred further that if $\left|P_{n}(x)\right| \leqq \mu$ for $a \leqq x \leqq b$, and if $a<a^{\prime}<b^{\prime}<b$, then

$$
\left|P_{n}^{\prime}(x)\right| \leqq C \mu n
$$

for $a^{\prime} \leqq x \leqq b^{\prime}$, where $C$ depends only on $a, b, a^{\prime}$, and $b^{\prime}$.

The immediate application of this result to the problem of convergence is not apparent. It is possible to reason further, however, as follows. Let it be supposed once more that the interval in question is $(-1,1)$. The function $P_{n}^{\prime}(\cos \theta)$, being a polynomial of degree $n-1$ at most in $\cos \theta$, is a cosine-sum of order $n-1$ at most in $\theta$. Since

$$
\cos k \theta \sin \theta=\frac{1}{2}[\sin (k+1) \theta-\sin (k-1) \theta]
$$

for all values of $k$, the expression

has the form

$$
Q_{n}(\theta)=\sin \theta P_{n}^{\prime}(\cos \theta)
$$

$$
Q_{n}(\theta)=A_{1} \sin \theta+A_{2} \sin 2 \theta+\cdots+A_{n} \sin n \theta .
$$

*S. Bernstein, loc. cit., pp. 6-11, proves this theorem arrectly, and makes it the basis for the discussion of the trigonometric theorem; but it is simpler to begin with de la Vallee Poussin's proof for the trigonometric case. 
Let the maximum of $\left|Q_{n}(\theta)\right|$ be denoted by $\mu_{1}$; the theorem of Bernstein for trigonometric sums is applicable again, with the conclusion that

$$
\left|Q_{n}^{\prime}(\theta)\right| \leqq \mu_{1} n
$$

for all values of $\theta$. As $Q_{n}(0)=0$, it follows that

$$
\left|Q_{n}(\theta)\right| \leqq \mu_{1} n|\theta| \text {. }
$$

Let $\theta$ have a value in the interval $\left(-\frac{1}{2} \pi, \frac{1}{2} \pi\right)$; then

and

$$
\left|\frac{\sin \theta}{\theta}\right| \geqq \frac{2}{\pi}>\frac{1}{2}, \quad|\theta| \leqq 2|\sin \theta|,
$$

$$
\left|\frac{Q_{n}(\theta)}{\sin \theta}\right| \leqq 2 \mu_{1} n \text {. }
$$

The same result can be obtained for $\pi / 2 \leqq x \leqq 3 \pi / 2$ from the relations

$$
\begin{gathered}
Q_{n}(\pi)=0, \quad\left|Q_{n}(\theta)\right| \leqq \mu_{1} n|\theta-\pi|, \\
|\theta-\pi| \leqq 2|\sin \theta| .
\end{gathered}
$$

The relation (4) therefore holds for all values of $\theta$. But

$$
\left|\frac{Q_{n}(\theta)}{\sin \theta}\right|=P_{n}^{\prime}(\cos \theta)=P_{n}^{\prime}(x),
$$

and, on the other hand, $\mu_{1} \leqq \mu n$. Hence it follows that*

$$
\left|P_{n}^{\prime}(x)\right| \leqq 2 \mu n^{2}
$$

for $-1 \leqq x \leqq 1$. If $\left|P_{n}(x)\right| \leqq \mu$ for $a \leqq x \leqq b$, the conclusion is that

$$
\left|P_{n}^{\prime}(x)\right| \leqq \frac{4 \mu n^{2}}{b-a}
$$

throughout the same interval.

Now let $f(x)$ be a given continuous function for $a \leqq x \leqq b$, let $m$ be a given fixed number $\geqq 1$, and let $P_{n}(x)$ be the polynomial of the $n$th degree or lower for which

$$
\int_{a}^{b}\left|f(x)-P_{n}(x)\right|^{m} d x
$$

is a minimum. For reasons analogous to those which appeared in connection with the trigonometric problem, if $p_{n}(x)$ is a polynomial of degree $n$ or lower, such that

$$
\left|f(x)-p_{n}(x)\right| \leqq \epsilon
$$

* This result is contained in a theorem of A. Markoff (see S. Bernstein, loc. cit., pp. 11-13), which states that $\left|P_{n}(x)\right| \leqq \mu n^{2}$ throughout the interval. If it could be shown above that $\left|Q_{n}(\theta) /(\sin \theta)\right| \leqq \mu_{1} n$, the present method would prove Markoff's theorem completely. 
throughout the interval, $\epsilon$ being small, the error of the approximating polynomial $P_{n}(x)$ for $f(x)$ is the same as the error of the approximating polynomial for

$$
\phi(x)=f(x)-p_{n}(x),
$$

and the latter is easier to deal with.

Suppose, then, that $\phi(x)$ is an arbitrary continuous function such that

$$
|\phi(x)| \leqq \epsilon
$$

throughout $(a, b)$. Let $\pi_{n}(x)$ be the approximating polynomial of the $n$th degree for $\phi(x)$, let $\mu_{n}=\left|\pi_{n}\left(x_{0}\right)\right|$ be the maximum of $\left|\pi_{n}(x)\right|$ in $(a, b)$, and let it be assumed for the moment that $\mu_{n} \geqq 4 \epsilon$.

In consequence of (5), if

$$
\left|x-x_{0}\right| \leqq \frac{b-a}{8 n^{2}}
$$

it will certainly be true that

$$
\begin{aligned}
\left|\pi_{n}(x)-\pi_{n}\left(x_{0}\right)\right| & \leqq \frac{1}{2} \mu_{n}, \\
\left|\pi_{n}(x)\right| & \geqq \frac{1}{2} \mu_{n}, \\
\left|\pi_{n}(x)-\phi(x)\right| & \geqq \frac{1}{4} \mu_{n} .
\end{aligned}
$$

The last relation holds throughout an interval of length at least $(b-a) /\left(8 n^{2}\right)$, and it is therefore certain that

$$
\gamma_{n}=\int_{a}^{b}\left|\pi_{n}(x)-\phi(x)\right|^{m} d x \geqq \frac{b-a}{8 n^{2}} \cdot\left(\frac{\mu_{n}}{4}\right)^{m} .
$$

On the other hand, $\dot{\gamma}_{n}$ is at any rate not greater than the value that would be obtained if $\pi_{n}(x)$ in the integral were replaced by zero, that is,

Hence

$$
\gamma_{n} \leqq(b-a) \epsilon^{m}
$$

$$
\begin{aligned}
\frac{(b-a)}{8 n^{2}} \cdot\left(\frac{\mu_{n}}{4}\right)^{m} & \leqq(b-a) \epsilon^{m}, \\
\mu_{n} & \leqq 4 \epsilon \cdot 8^{1 / m} n^{2 / m} .
\end{aligned}
$$

The last relation has been obtained on the hypothesis that $\mu_{n} \geqq 4 \epsilon$, but it is clearly satisfied also if $\mu_{n}<4 \epsilon$.

Let $\rho_{n}$ be the maximum of $\mid \pi_{n}(x)-\phi(x)^{\mid}$; then $\rho_{n} \leqq \mu_{n}+\epsilon$, while, at the same time, if $\phi(x)$ has the form $f(x)-p_{n}(x), \rho_{n}$ is the maximum of the difference between $f(x)$ and its approximating polynomial $P_{n}(x)$. Thus the following assertion is justified:

If $f(x)$ is continuous for $a \leqq x \leqq b$, and can be represented throughout the intercal by a polynomial of degree $n$ or lower with an error not exceeding $\epsilon_{n}$, if 
$P_{n}(x)$ is the approximating polynomial of degree $n$ for $f(x)$, corresponding to the exponent $m$, and if $\rho_{n}$ is the maximum of $\left|f(x)-P_{n}(x)\right|$, then

$$
\rho_{n} \leqq c_{m} n^{2 / m} \epsilon_{n}
$$

where $c_{m}$ is a constant depending only on $m$.

The constant $c_{m}$ corresponds to that which was denoted by $k_{m}+1$ in the trigonometric case; the difference in notation has no significance:

The polynomial $P_{n}(x)$ will converge uniformly to the value $f(x)$ for $a \leqq x \leqq b$, if it is possible to make $\epsilon_{n}$ take on such values that

$$
\lim _{n=\infty} \sqrt[m]{n^{2}} \cdot \epsilon_{n}=0
$$

Let $\omega(\delta)$ be the modulus of continuity of $f(x)$, as before. There will exist polynomial representations* of $f(x)$ such that $\epsilon_{n} \leqq c^{\prime} \omega[(b-a) / n]$, where $c^{\prime}$ is independent of $n$, for all positive integral values of $n$. A sufficient condition for uniform convergence throughout the interval $a \leqq x \leqq b$ is that $\lim _{\delta=0} \omega(\delta) / \sqrt[m]{\delta^{2}}=0$.

For $m \leqq 2$, the condition just stated would require that $f(x)$ be a constant, but it is easy to replace this condition by a more satisfactory one. If $1<m \leqq 2$, let it be assumed that $f(x)$ possesses a continuous first derivative having the modulus of continuity $\omega_{1}(\delta)$. It is possible to make $\dagger$

$$
\epsilon_{n} \leqq \frac{c^{\prime \prime}}{n} \omega_{1}\left(\frac{b-a}{n-1}\right)
$$

where $c^{\prime \prime}$, once more, is independent of $n$. For $1<m \leqq 2$, a sufficient condition for uniform convergence throughout the interval $a \leqq x \leqq b$ is that $f(x)$ have a continuous derivative, and that $\lim _{\delta=0} \omega_{1}(\delta) / \delta^{(2 / m)-1}=0$, where $\omega_{1}(\delta)$ is the modulus of continuity of the derivative. In case $m=2$, the condition thus obtained is simply that $f(x)$ have a continuous first derivative.

For $m=1$, a sufficient condition is that $f(x)$ have a continuous second derivative. $\ddagger$

If $f(x)$ possesses a greater degree of regularity than is required for mere convergence, theorems on the degree of convergence of the approximating polynomials can be written down at once; it is not necessary to dwell on the point further.

6. Polynomial case, end-points excluded. The relation (6) once having been obtained, it is possible to make use of (3) to some advantage in connection with the convergence at interior points of the interval.

* See, e.g., D. Jackson, these T r a $\mathrm{n}$ s a c $\mathrm{t}$ i o $\mathrm{n} \mathrm{s}$, vol. 14, as already cited, pp. 353-354.

$\dagger$ D. Jackson, these T r a n s a $t$ i o $\mathrm{n}$ s, vol. 14, as cited, p. 354.

†Cf. preceding citation. 
Let the notation leading up to (6) be kept in force, let $a^{\prime}$ and $b^{\prime}$ be two numbers such that

$$
a<a^{\prime}<b^{\prime}<b,
$$

and let $\mu_{n}^{\prime}=\left|\pi_{n}\left(x_{1}\right)\right|$ be the maximum of $\left|\pi_{n}(x)\right|$ for $a^{\prime} \leqq x \leqq b^{\prime}$, with the assumption, temporarily, that $\mu_{n}^{\prime} \geqq 4 \epsilon$.

By (6) and (3),

$$
\left|\pi_{n}(x)-\pi_{n}\left(x_{1}\right)\right| \leqq 4 C \epsilon \cdot 8^{1 / m} n^{(2 / m)+1}\left|x-x_{1}\right|=C^{\prime} \epsilon \cdot n^{(2 / m)+1}\left|x-x_{1}\right|,
$$

as long as $x$ is in $\left(a^{\prime}, b^{\prime}\right)$; the constant $C^{\prime}$ depends only on $m, a, b, a^{\prime}$, and $b^{\prime}$. If

$$
\left|x-x_{1}\right| \leqq \frac{\mu_{n}^{\prime}}{2 C^{\prime} \epsilon \cdot n^{(2 / m)+1}},
$$

$x$ will surely be in $\left(a^{\prime}, b^{\prime}\right)$, on one side of $x_{1}$ at least, for $n \geqq n_{0}\left(m, a, b, a^{\prime}, b^{\prime}\right)$, because the right-hand member of (7), in consequence of (6) and the fact that $\mu_{n}^{\prime} \leqq \mu_{n}$, can not exceed $2 \cdot 8^{1 / m} /\left(C^{\prime} n\right)$; and it will follow further that

$$
\begin{aligned}
\left|\pi_{n}(x)-\pi_{n}\left(x_{1}\right)\right| & \leqq \frac{1}{2} \mu_{n}^{\prime}, \\
\left|\pi_{n}(x)\right| & \geqq \frac{1}{2} \mu_{n}^{\prime}, \\
\left|\pi_{n}(x)-\phi(x)\right| & \geqq \frac{1}{4} \mu_{n}^{\prime} .
\end{aligned}
$$

These relations hold throughout an interval, the length of which is at least equal to the right-hand member of (7).

This means that

$$
\gamma_{n}=\int_{a}^{b}\left|\pi_{n}(x)-\phi(x)\right|^{m} d x \geqq \frac{\mu_{n}^{\prime}}{2 C^{\prime} \epsilon \cdot n^{(2 / m)+1}} \cdot\left(\frac{\mu_{n}^{\prime}}{4}\right)^{m} .
$$

But it is still true that

Hence

$$
\gamma_{n} \leqq(b-a) \epsilon^{m}
$$

$$
\begin{aligned}
\therefore \ell_{n}^{\prime m+1} & \leqq C^{\prime \prime} n^{(2 / m)+1} \epsilon^{m+1}, \\
\mu_{n}^{\prime} & \leqq C^{\prime \prime \prime} \epsilon \cdot n^{(m+2) /[m(m+1)]},
\end{aligned}
$$

the coefficients $C^{\prime \prime}$ and $C^{\prime \prime \prime}$, like the preceding $C^{\prime}$ s, depending only on $m, a$, $b, a^{\prime}$, and $b^{\prime}$. A relation of the same form continues to hold if $\mu_{n}^{\prime}<4 \epsilon$.

There is perhaps no need of setting down at length the resulting theorems on convergence and degree of convergence in the interior of the original interval. The results of the section are summed up by saving that the exponent $1 / m$ of the trigonometric case, which became $2 / m$ in the preceding section, is replaced here by

$$
\frac{1}{m} \cdot \frac{m+2}{m+1} .
$$

The University of Minnesota, Minneapolis, Minn. 